Background The identification of biomarkers predicting a better outcome to adoptive $\mathrm{T}$ cell therapy is an emerging, still nascent field. We previously completed a phase 2 clinical trial of autologous adoptive EBV-specific cytotoxic T lymphocyte (CTL) immunotherapy following gemcitabine + carboplatin chemotherapy as first line treatment in 35 advanced incurable stage 4c nasopharyngeal carcinoma (NPC) patients. Here, we aim 1) to evaluate exosome proteins for potential specific predictive biomarkers of benefit to adoptive $\mathrm{T}$ cell therapy and 2) to investigate if a specific immunophenotype of our generated EBV targeting CTLs correlates with survival benefit.

Methods We isolated exosome from plasma samples by size exclusion chromatography and magnetic-based isolation technology, followed by fluorescence-activated cell sorting (FACS) analysis, Western blot analysis for immune-related checkpoint molecules, or mass spectrometry for exosomal peptide detection. All the generated CTLs were analysed by gene expression microarray as well as a FACS system with the use of $\mathrm{T}$ cell-specific 23-antibody panel for comprehensive immunophenotyping. The representative CTL samples were also subject to single-cell (sc) RNA-Seq with DNA barcoded antibodies for comprehensive integrated transcriptomics and immunophenotyping analysis.

Results Patients with overall survival longer than 2 years were grouped as long survivors and the remaining patients were grouped as short survivors. Differentially expressed immune-related checkpoint molecules, such as PD1, ICOS, and CD137, were detected in in pre-treatment exosome of long and short survivors. Furthermore, more than 13,000 high confident and unique peptides which belong to 1,500 unique proteins were identified and quantified by mass spectrometry from the purified plasma exosome. Pathway enrichment analysis further showed that plasma exosome of the short survivors had significantly higher innate immune response-activating signal transduction-related peptides detected ( $p$-value $=4.81 \times 10-5)$. The transcriptomic analysis revealed that statistically lower expressions of SELL (CD62L) and LEF1 were found in the EBV CTL of the short survivors, suggesting that the EBV CTL of short survivors were characterized by a lesser central memory $\mathrm{T}$ cell phenotype.

Conclusions Our data reports that specific pre-treatment plasma exosome proteins, and separately, transcriptomic profile of the generated baseline EBV CTLs prior to infusion into the patients correlate with patient survival, suggesting that they could be used together as biomarkers to predict outcome in the advanced NPC patients treated with this chemo-immunotherapy combination. More in-depth analysis of the immunophenotyping of all the CTLs and the representative CTLs' scRNA-Seq data will be presented at the meeting.

Acknowledgements This work was supported by Singapore government funding (National Medical Research Council (NMRC) - Open Fund - Large Collaborative Grant (OF-LCG) and National Cancer Centre Singapore Block Grant as well as in part by Tessa Therapeutics Ltd.
TRANSCRIPTOMIC ANALYSIS OF DYSFUNCTIONAL CD8+ TILS IN MELANOMA IDENTIFIES BILE ACID AND MTOR PATHWAYS AS NOVEL POTENTIAL IMMUNOTHERAPY TARGETS

${ }^{1}$ Cheryl Cameron, 'Brian Richardson, 'Jackelyn Golden, ${ }^{2}$ Lukas Pfannensttiel, ${ }^{1}$ Michael Cartwright, ${ }^{1}$ Yousef Moustafa, ${ }^{2}$ Samjhana Thapaliya, ${ }^{2}$ Gustavo Roversi, ${ }^{2}$ Yee Peng Phoon, ${ }^{1}$ Mark Cameron*, ${ }^{2}$ Brian Gastman*, ${ }^{1}$ Cheryl Cameron. ${ }^{1}$ Case Western Reserve University, Cleveland, $\mathrm{OH}, \mathrm{USA} ;{ }^{2}$ Cleveland Clinic, Cleveland, $\mathrm{OH}, \mathrm{USA}$

\subsection{6/LBA2019.6}

Background Coexpression of the immune checkpoint receptors PD-1 and TIM-3 is associated with dysfunction of tumor infiltrating lymphocytes in melanoma (MEL) and squamous cell carcinoma (SCC). To identify the mechanisms underlying this dysfunctional phenotype and to identify targets to rescue immune function, we performed transcriptional profiling of PD-1+TIM-3+CD8+ TILs sorted directly from MEL and SCC tumors in conjunction with immunologic phenotyping. We identified a number of novel dysregulated pathways and associated gene signatures in the PD-1+TIM- + subset that have not previously been reported in TILs, including bile acid metabolism, and demonstrated that these pathways are highly correlated not only to immune checkpoint receptor expression but also to MTOR pathway activation.

Methods Surgically excised melanoma tumors were dissociated and mononuclear cells were isolated by Ficoll gradient separation and cryopreserved. For sorting based on immune checkpoint receptor expression, a cocktail of monoclonal antibodies to the following targets, including viability dye was used: CD3, CD4, CD8, CD45RA, CD27, CCR7 PE, CD14, CD19, Live/Dead, BTLA, TIM-3, PD-1, CTLA-4, TIGIT and LAG-3. RNA was purified from 10,000 sorted cells using RNeasy Mini Kits (Qiagen), followed by RNASeq library generation using TruSeq Stranded Total RNA HT Kits (Illumina). Gene set variation analysis (GSVA) was used for pathway analysis, as well as linear regression modelling using limma (Bioconductor).

Results Transcriptomic analysis of CD8+ TILs revealed 4,228 genes $(\mathrm{P}<0.05$, t-test $)$ as differentially expressed in TILs vs. functional peripheral CD8 $\mathrm{T}$ cells. Using a nonlinear dimensionality-reduction technique, we found that there were several unique clusters of cell surface marker expression that were present at significantly different frequencies in the TILs. We used pathway enrichment analysis and linear regression modeling to identify gene signatures that correlate with the progressive and coordinate expression of PD-1, TIM-3, and additional checkpoint receptors thereby driving progressive dysfunction on in TILs. The peroxisome and bile acid metabolism pathways were significantly enriched in the TIL transcriptome. Moreover, higher frequencies of events in dysfunctional clusters were strongly correlated with positive enrichment of the bile acid metabolism pathway and enhanced MTOR signaling.

Conclusions Transcriptomic analysis of PD-1+TIM-3+ CD8+ TILs identified novel candidate mechanisms of immune dysfunction in CD8 + TILs from patients with metastatic melanoma who fail immunotherapy. Our study identifies the bile 
acid and MTOR metabolic pathways as a potential novel therapeutic targets for complementary therapy to restore immune function in melanoma and SCC patients.

Ethics Approval This study was performed under an IRB approved protocol.

\section{P853 SINGLE CELL TRANSCRIPTOME ANALYSIS IDENTIFIES UNIQUE FEATURES IN CIRCULATING CD8+ T CELLS THAT CAN PREDICT IMMUNOTHERAPY RESPONSE IN MELANOMA PATIENTS}

${ }^{1}$ Chuan Li, ${ }^{2}$ Yee Peng Phoon, ${ }^{1}$ Keaton Karlinsey, ${ }^{2}$ Ye Tian, ${ }^{2}$ Samjhana Thapaliya, ${ }^{1}$ Lili Qu, ${ }^{3}$ Mark Cameron, ${ }^{3}$ Cheryl Cameron, ${ }^{1}$ Antoine Menoret, ${ }^{2}$ Pauline Funchain, ${ }^{2}$ Jung-Min Song, ${ }^{2} \mathrm{C}$ Marcela Diaz-Montero, ${ }^{1}$ Annabelle Rodriguez, ${ }^{1}$ Christopher Bonin, ${ }^{1}$ Anthony Vella, 'Beiyan Zhou*, 'Brian Gastman*. 'University of Connecticut, Farmington, CT, USA; ${ }^{2}$ Cleveland Clinic, Cleveland, $\mathrm{OH}$, USA; ${ }^{3}$ Case Western Reserve University, Cleveland, $\mathrm{OH}$, USA

\subsection{6/LBA2019.7}

Background Immune checkpoint blockade (ICB) has greatly advanced the treatment of melanoma. A key component of ICB is the stimulation of CD8 + T cells in the tumor. However, ICB therapy only benefits a subset of patients and a reliable prediction method that does not require invasive biopsies is still a major challenge in the field.

Methods We conducted a set of comprehensive single-cell transcriptomic analyses of CD8 $+\mathrm{T}$ cells in the peripheral blood (mPBL) and tumors (mTIL) from 8 patients with metastatic melanoma.

Results Compared to circulating CD8 $+\mathrm{T}$ cells from healthy donors (hPBL), mPBLs contained subsets resembling certain features of mTIL. More importantly, three clusters (2, 6 and $15)$ were represented in both mPBL and mTIL. Cluster 2 was the major subset of the majority of hPBL, which phenocopied hallmark parameters of resting T cells. Cluster 6 and 15 were uniquely presented in melanoma patients. Cluster 15 had the highest PD-1 levels, with elevated markers of both activation and dysfunction/exhaustion; while Cluster 6 was enriched for 'dormant' cells with overall toned-down transcriptional activity except PPAR signaling, a known suppressor for $\mathrm{T}$ cell activation. Interestingly, unlike other mTIL clusters that would classically be defined as exhausted, Cluster 15 exhibited the highest metabolic activity (oxidative-phosphorylation and glycolysis). We further analyzed total sc-transcriptomics using cell trajectory algorithms and identified that these three clusters were the most distinct subtypes of CD8 $\mathrm{T}$ cells from each other, representing: resting (cluster 2), metabolically active-dysfunctional (cluster 15), and dormant phenotypes (cluster 6). Further, three unique intracellular programs in melanoma drive the transition of resting CD8 $+\mathrm{T}$ cells (cluster 2) to both metabolic/dysfunctional (cluster 15) and dormant states (cluster 6) that are unique to tumor bearing conditions. Based on these high-resolution analyses, we developed original algorithms to build a novel ICB response predictive model using immune-blockade co-expression gene patterns. The model was trained and tested using previously published GEO datasets containing CD8 $\mathrm{T}$ cells from anti-PD-1 treated patients and presented an AUC of 0.82 , with $92 \%$ and $89 \%$ accuracy of ICB response in the two datasets.
Conclusions We identified and analyzed unique populations of $\mathrm{CD} 8+\mathrm{T}$ cells in circulation and tumor using high-resolution single-cell transcriptomics to define the landscape of CD8 $+\mathrm{T}$ cell states, revealing critical subsets with shared features in PBLs and TILs. Most importantly, we established an innovative model for ICB response prediction by using peripheral blood lymphocytes.

Ethics Approval This study was performed under an IRB approved protocol.

\section{Checkpoint blockade therapy}

\section{P854 CONSTRUCTION OF THE IMMUNE LANDSCAPE OF DURABLE RESPONSE TO CHECKPOINT BLOCKADE THERAPY BY INTEGRATING PUBLICLY AVAILABLE DATASETS}

${ }^{1}$ Nils-Petter Rudqvist* ${ }^{2}{ }^{2}$ Roberta Zappasodi, ${ }^{3}$ Daniel Wells, ${ }^{4}$ Vésteinn Thorsson, ${ }^{5}$ Alexandria Cogdill, ${ }^{6}$ Anne Monette, ${ }^{7}$ Yana Najjar, ${ }^{8}$ Randy Sweis, ${ }^{9}$ Erik Wennerberg, ${ }^{10}$ Praveen Bommareddy, ${ }^{5}$ Cara Haymaker, ${ }^{11}$ Uqba Khan, ${ }^{12}$ Heather McGee, ${ }^{2}$ Wungki Park, ${ }^{13}$ Houssein Abdul Sater, ${ }^{3}$ Christine Spencer, ${ }^{14}$ Nicholas Tschernia, ${ }^{15}$ Maria Ascierto, ${ }^{16}$ Valentin Barsan, ${ }^{17}$ Vinita Popat, ${ }^{18}$ Sara Valpione, ${ }^{19}$ Benjamin Vincent*. ${ }^{1}$ Weill Cornell Medicine, New York, NY, USA; TimIOs Lead, New York, NY, USA; ${ }^{2}$ Memorial Sloan Kettering Cancer Center, New York, NY, USA; Parker Institute of Cancer Immunotherapy, San Francisco, CA, USA; ${ }^{3}$ Parker Institute of Cancer Immunotherapy, San Francisco, CA, USA; ${ }^{4}$ Institute for Systems Biology, Seattle, WA, USA; ${ }^{5}$ The University of Texas MD Anderson Cancer Center, Houston, TX, USA; ${ }^{6}$ McGill University, Montréal, Québec, Canada; ${ }^{7}$ University of Pittsburgh, Pittsburgh, PA, USA; ${ }^{8}$ University of Chicago, Chicago, IL, USA; ${ }^{9}$ Weill Cornell Medicine, New York, NY, USA; ${ }^{10}$ Replimune, Woburn, MA, USA; ${ }^{11}$ Weill Cornell Medicine, New York, NY, USA; Memorial Sloan Kettering Cancer Center, New York, NY, USA; New York Presbyterian Brooklyn Methodist Hospital, New York, NY, USA; ${ }^{12}$ Icahn School of Medicine at Mount Sinai, New York, NY, USA; ${ }^{13}$ National Cancer Institute, Bethesda, MD, USA; ${ }^{14}$ University of North Carolina, Chapel Hill, NC, USA; ${ }^{15}$ AstraZeneca, Gaithersburg, MD, USA; ${ }^{16}$ Stanford University School of Medicine, Stanford, CA, USA ${ }^{17}$ University of Texas Southwestern Medical Center, Dallas, TX, USA; ${ }^{18}$ Cancer Research UK Manchester Institute, Manchester, UK; ${ }^{19}$ University of North Carolina, Chapel Hill, NC, USA

\subsection{6/LBA2019.8}

Background Immune checkpoint blockade (ICB) has revolutionized cancer treatment. However, long-term benefits are only achieved in a small fraction of patients. Understanding the mechanisms underlying ICB activity is key to improving the efficacy of immunotherapy. A major limitation to uncovering these mechanisms is the limited number of responders within each ICB trial. Integrating data from multiple studies of ICB would help overcome this issue and more reliably define the immune landscape of durable responses. Towards this goal, we formed the TimIOs consortium, comprising researchers from the Society for Immunotherapy of Cancer Sparkathon TimIOs Initiative, the Parker Institute of Cancer Immunotherapy, the University of North Carolina-Chapel Hill, and the Institute for Systems Biology. Together, we aim to improve the understanding of the molecular mechanisms associated with defined outcomes to ICB, by building on our joint and multifaceted expertise in the field of immuno-oncology. To determine the feasibility and relevance of our approach, we have assembled a compendium of publicly available gene expression datasets from clinical trials of ICB. We plan to analyze this data using a previously reported pipeline that 